To appear in:

McKay Illari, P., Russo, F., and J. Williamson (eds.): Causality in the Sciences, Oxford University Press 2011.

\title{
Mechanisms in Dynamically Complex Systems
}

\author{
M. Kuhlmann \\ Institute for Philosophy, University of Bremen, Germany
}

\section{Introduction}

In recent debates mechanisms are often discussed in the context of 'complex systems', with certain biological examples, for instance concerning biochemical processes, as paradigmatic cases. Complex systems of this kind often have a complicated compositional structure. I want to draw the attention to the fact that there is still another, radically different kind of complex system, in fact one that many scientists - in particular in the physical sciences - regard as the only genuine kind of complex system. Instead of being compositionally complex these systems rather exhibit highly nontrivial dynamical patterns, on the basis of structurally simple arrangements of large numbers of non-linearly interacting constituents. To be sure, I want to call this kind 'dynamically complex systems'. The characteristic dynamical patterns in dynamically complex systems arise from the interaction of the system's parts largely irrespective of many properties of these parts. One example, which has been studied extensively in statistical physics, is the ferromagnet with a surprising dynamical behaviour despite of the fact that it consists of nothing more than a simple array of numerous identical dipoles. Analyses of dynamically complex systems are by no means limited to physics. For instance, it is common practice to model socio-economic contexts by using dynamical multi-agent systems, which deal with 'microscopic' agents with a very simple individual behaviour in a very simple arrangement.

Whereas for a compositionally complex system it is usually feasible to predict its behaviour once the compositional structure and the behaviour of its parts is known, this is completely different in the case of dynamically complex systems. Here the knowledge of the compositional structure, e.g. agents with only two possible actions 
arranged on a square lattice, together with the knowledge of the behaviour of its parts in isolation as well as in simple composites, often allows for hardly any straightforward predictions of the dynamical behaviour of a given complex system. Nevertheless, an ensemble of similar complex systems can exhibit surprising statistical characteristics, the robustness of which calls for an explanation in terms of underlying generating mechanisms. Thus, not only for compositionally complex systems, but also for dynamically complex systems, the identification of generating mechanisms is essential in order to explain their often surprising behaviour.

However, I want to argue, dynamically complex systems are not sufficiently covered by the available conceptions of mechanisms. Whereas for mechanisms in compositionally complex systems the decomposition into modules is an essential and non-trivial task, it is usually largely a non-issue for dynamically complex systems. Instead, the recognition and detailed (statistical) analysis of dynamical patterns that are to be explained becomes one main task, besides the identification of generating mechanisms. The most important novelty in dynamically complex systems is the fact that the material nature of the mechanisms' parts in dynamically complex systems is irrelevant in a far more drastic way than in many classical biological mechanisms, for instance. Structurally similar dynamical patterns can occur in materially completely diverse phenomena such as traffic jams, avalanches, earthquakes, tsunamis and financial market crashes. In each of these cases one has a system with a large number of elements, which displays a surprising macroscopic behaviour that results purely from the local interaction of the system's components. Due to the resulting predominance of structural over material considerations in complex systems research, which is underlined by the formation of numerous interdisciplinary projects and even whole scientific fields, mechanisms in dynamically complex systems must be construed in a more abstract structural fashion. ${ }^{1}$ Despite these and other differences, it is still appropriate to talk about 'mechanisms' both for compositionally as well as for dynamically complex systems, since, among other things, in both cases the interaction of parts and the robustness regarding the resulting behaviour of the composite system are essential, albeit these features need to be filled in a different way.

Many widely used notions in complex systems research, such as complexity, emergence and mechanisms, are notoriously and to some extent inevitably vague. Among other things this vagueness manifests itself in various lists, e.g. of emergent phenomena or nonlinear mechanisms, whose items are neither on the same level nor situated in any clear conceptual hierarchy. It is one of my aims in this paper to go some way towards a clarification, either by characterising some notions or by highlighting

\footnotetext{
${ }^{1}$ In short, I use the term 'structural' in the philosophy of physics fashion (structural versus material) and not the philosophy of biology one (structural versus functional). For philosophers of biology, 'functional descriptions' abstract from everything other than input-output relations or the function that a part plays in a given whole, whereas 'structural descriptions' go beyond functional ones by referring to the inner material structure that underlies or may underlie a given functional relation. In contrast to that, the philosophy of physics' usage of 'structural' refers to abstracting from any particular material entities. This usually means that one focuses on abstract mathematical structures, such as the harmonic oscillator or certain symmetries or dynamical patterns like bifurcations in chaotic systems. In physical contexts the focus on abstract mathematical structures often reveals decisive insights into essential features of a physical system that are invisible with a detailed material description.
} 
certain hidden ambiguities. My main goal is to explore whether and how the notion of a mechanism has to be modified in the case of mechanisms in (dynamically) complex systems. Moreover, I will show under which conditions the widespread, if not inflationary talk about mechanisms in (dynamically) complex systems stretches the notion of mechanisms beyond its reasonable limits and is no longer legitimate. Thus, I want to explore the boundary of the notion of mechanisms by giving reasons for distinguishing warranted from unwarranted claims about successful mechanistic explanations. To this end I will carve out a minimal notion of mechanisms that allows theorists, first, to incorporate many complex systems analyses into the mechanistic program and, second, to say in which cases claims for a mechanistic explanation are at least premature. I will present two detailed examples, one of which I see on the (yet) unwarranted and the other one on the warranted side of the boundary of a minimal notion of mechanisms.

\section{Dynamical Complexity}

\subsection{Compositional versus Dynamical Complexity}

The most important distinction in my analysis is that between compositional and dynamical complexity. What I call compositional complexity is also discussed under the labels structural, combinatorial or detail complexity. ${ }^{2}$ Alternatively, one could also talk about set-up complexity because the complexity is due to a complicated organisation of the set-up. Note that my usage of the term 'compositional complexity' should not be understood in the sense of complex rules of composition, which play a role in the discussion of emergence. ${ }^{3}$ The compositionally complex systems I have in mind are typically linear systems which obey the principle of superposition, i.e. the behaviour of the compound system is the summation of the behaviours of its component parts in the sense that the system behaviour can be predicted by the traditional reductionist procedure of identifying components and characterising their individual input-output behaviours. In compositionally complex systems the complexity resides in the large number of relevant variables that characterise the component parts together with the detailed organisation which is one out of very many possible combinations of the component parts. I call such a system compositionally complex because the individual behaviours of its parts and the detailed way how these parts are organized in the compound system are decisive for the overall system behaviour. Change the behaviour or the input of one of its parts or change the relation of two parts and you will in general change the behaviour of the whole system. Thus in compositionally complex systems many micro details have a measurable (linear) effect on the studied behaviour of the whole system.

In contrast, for dynamically complex systems very few parameters are usually sufficient to describe the behaviour of the whole system one is interested in. In most

\footnotetext{
${ }^{2}$ For instance, see Sterman (2000: 21) and Érdi (2007: 1). Moreover, I should point out that there is also a divergent technical notion of 'compositional complexity' as "a measure of bias in the sequence composition" (Zvelebil/Baum 2007: 151), which is used in particular in the analysis of DNA sequences.

${ }^{3}$ See Hüttemann (2004: chapter 3) and Hüttemann/Terzidis (2000).
} 
cases the vast majority of micro details is irrelevant, in the sense that a change of most microscopic variables as well as a change of most interrelations of the component parts will have no effect at all on the overall system behaviour. ${ }^{4}$ Dynamical complexity is characterised by the fact that even in compositionally simple systems with simple (but nonlinear) rules that determine the dynamics the resulting time series can be unexpectedly complex. For instance, a nonlinear double pendulum, i.e. a pendulum with another pendulum attached to it, exhibits complex chaotic behaviour, due to the nonlinearity of the rules that determine its dynamics, while the compositional complexity is as low as one can think. Thus, dynamical complexity arises from the nonlinear interactions of the subunits over time.

The example of the double pendulum allows me to forestall a possible misunderstanding. Dynamically complex systems also do have components, no less than compositionally complex systems. However, most facts about the nature of these components as well as their initial arrangement have no bearing on the complex system behaviour one wants to explain. In the case of the double pendulum, for instance, it makes no difference for the complexity of its behaviour how long the two pendulums are, out of which material they are made and in which initial state they are arranged. To put it another way, dynamically complex systems don't need to look differently from compositionally complex systems. They may still have recognizable components which behave and interact in a regular fashion and thereby give rise to a particular behaviour of the whole system. However, knowledge about the detailed nature of these components and the way how they are organized in the whole does not render the complexity of the system behaviour understandable.

Strictly speaking, a system exhibits either compositional or dynamical complexity only with respect to a certain behaviour to be explained. That is, one and the same system can be compositionally complex with respect to the behaviour of, e.g., one quantity and dynamically complex with respect to the behaviour of another quantity, or not complex at all with respect to the behaviour of still another quantity. For instance, the community of financial market traders may be compositionally complex with respect to the money they spend on travelling, dynamically complex with respect to the stock market prices they generate and not complex at all with respect to their collective weight. The dependence of complexity on the particular quantity or phenomenon one is studying is reflected by the fact that the issue of compositional versus dynamical complexity as well as the issue of mechanisms in such systems play a major role in the broader context of explanations. And the quality of an explanation in turn depends on that aspect of a phenomenon one seeks to understand. Thus complexity, mechanisms and explanations are pragmatic matters, which depend decisively on one's explanatory interests.

\footnotetext{
${ }^{4}$ The occurrence of the so-called butterfly effect, a well-known characteristic feature of dynamically complex systems is not in conflict with how I characterised dynamical complexity and explains the addition 'In most cases'. In some cases, tiny variations of the initial conditions are sufficient to generate a drastic effect for the whole system. But even this effect does not occur for compositionally complex systems where, due to their linearity, similar variations of the initial conditions always lead to similar effects for the whole system.
} 
Talk about 'dynamical complexity' in complex systems' research has a certain further ambiguity since the term refers to two intimately connected but still different perspectives, strictly speaking. The first meaning is dynamically emerging complexity, i.e. an unpredictable complexity in the system behaviour that arises while the system evolves in time, although the rules for the interactions between its components are very simple (albeit nonlinear). The second meaning is complexity displayed in the statistical characteristics of the dynamics, i.e. it refers to a complex measurable phenomenology of the dynamics. Roughly the ambiguity concerning 'dynamical complexity' is the difference between process and result. Examples for such a complex coming about are the endogenous formation of abrupt changes and extreme events through the nonlinear interaction of the system's subunits without an abrupt or extreme external influence. An example for a 'complex result' is statistical self-similarity, e.g., of the fluctuations of some quantity (see below). If some given dynamics is statistically self-similar, it is often referred to as 'fractal dynamics'. But of course, while the system evolves, fractality cannot be recognized. The fractality involved here shows up only in the statistical analysis of the data set of the whole time series, which results from the dynamics of the system. To a certain degree the ambiguity of 'dynamical complexity' is already inherent in the term 'dynamics', which is often referred to almost as an object, whereas the paraphrase of 'dynamics' as the 'evolution of a system in time' exhibits the procedural character.

Dynamical complexity in the first sense emerges only in the temporal evolution, i.e. in the dynamics of a compound system without any need for complex initial conditions. In other words, even if the set-up of the system is very simple, its dynamics can exhibit an unpredictable complexity. In addition, the composition of the system may, in concrete cases, also be complicated or, if one wishes to say so, 'complex', but this compositional complexity is not responsible for the dynamically emerging complexity I am addressing. In order to isolate and understand how complexity arises it is therefore advisable to make the assumptions about the initial set-up of the system as simple as possible. Although some kind of dynamical complexity in the second sense, i.e. a complex statistical phenomenology of the dynamics, could in principle result from the compositional complexity of the initial set-up or complex influences from the system's environment, there are very powerful and subtle methods for discriminating complex statistical characteristics that most likely emerged only in the temporal evolution of compositionally simple systems. One of the main reasons behind this assessment is the experience with other systems that are well understood and where a complex dynamical phenomenology emerged purely endogenously by the (nonlinear) interaction of the system's otherwise simple subunits.

\subsection{Dynamical Complexity: From Data Analysis to Mechanisms}

Often, significant complex dynamical patterns are very difficult to identify because they are hidden beneath other regular or random processes (see the 'DFA method' below). Since the non-trivial identification of certain characteristic features in the dynamics of a system is taken as a strong indicator for corresponding underlying 'mechanisms', it becomes hard to disentangle description and analysis on the one side and explanation on 
the other side. Interestingly, Goldberger (2006), for instance, makes no clear distinction between phenomena (which are to be explained) and mechanisms (on which the explaining is usually taken to rest) when he presents his list of 'nonlinear/complexityrelated mechanisms and phenomena in physiology'. Since, on the one hand, dynamically emerging complexity necessarily results in complexity displayed in the statistical characteristics of the dynamics and, on the other hand, the occurrence of such statistical characteristics is in turn the most important indication of underlying dynamically emerging complexity (of the corresponding kind), these two aspects are often identified without further reflection about their difference.

As one can see in the later examples, the ambiguity of the expression 'dynamical complexity' (i.e. process versus result) is, to a certain degree, transferred to the way the term 'mechanism' is used in complex systems' research. For instance, there is, side by side, talk about 'feedback mechanisms' and 'fractal mechanisms', although these two issues, feedback and fractality, are not on the same conceptual level. Whereas feedback is a process that can occur in the evolution of a system, fractality is (in this context) a characteristic property of the statistics of a time series. Thus, strictly speaking, fractality itself is not a process in time at all - although it may be a strong indicator for a certain underlying process or mechanism that generates fractality. I can see two options now. Either one 'simply' points out that the expression 'mechanism' is inappropriate in such cases. Or one explores under which conditions one can make sense of this common use. I will go for the second option.

As mentioned above, investigations of dynamically complex systems are-due to the crucial role of structural considerations - often pursued in interdisciplinary research groups. Econophysics, for instance, is a relatively young special science between physics and economics that tries to analyze and explain economical phenomena by using models, techniques and analytical tools from physics. ${ }^{6}$ Although the possibility of econophysics first appears puzzling, it is 'simply' grounded on the insight that important properties of, e.g., financial markets can be understood if one adopts a complex systems framework. The same reasoning stands behind many other, mostly computer-aided analyses of, e.g., traffic flow, opinion dynamics, social networks, avalanches, earthquakes, turbulences, tsunamis etc., and in a more general fashion in chaos theory, game theory or the theory of self-organisation. In these diverse contexts one observes similar dynamical patterns, which is seen as an indication that they are generated by structurally similar mechanisms. For this reason, it is often possible to use the same methods, models and analytical strategies, many of which were first devised in physics. Although in the case of econophysics the use of analytical tools from physics is particularly dominant, the general reasoning, the concepts, and the strategies are very similar in various other investigations of complex systems. Outstanding examples for the success of econophysics are the analysis and description of financial market crashes

\footnotetext{
${ }^{5}$ Among other things, Goldberger's list (p. 469) contains abrupt changes (e.g. bifurcations, intermittency and other bursting behaviours, bistability/multistability, phase transitions), complex periodic cycles and quasiperiodicities, nonlinear oscillations (e.g. limit cycles, phase-resetting, entrainment phenomena, pacemaker annihilation) and scale-invariance (diffusion limited aggregation, fractal and multifractal scaling, long-range correlations, self-organized criticality).

${ }^{6}$ See, for instance, Mantegna and Stanley (2000) and Johnson et al. (2003).
} 
by using the advanced physical theory of phase transitions, where the common characteristic is a sudden occurrence of a comprehensive change of the state of affairs.

Scale-invariance/self-similarity, power-law behaviour and the closely connected occurrence of 'universal behaviour' and criticality are important indications that one is dealing with a dynamically complex system. The existence of long-range correlations in fluctuating quantities is particularly interesting because it indicates that there may be an underlying long-memory process, i.e. that the fluctuation at a given time depends on what has happened at earlier times. By contrast, a Gaussian random walk process exhibits no long-range correlations because each change of the respective quantity is an independent event, so to say, which is not affected by previous changes. Long-range correlations are an implication of statistical self-similarity, which in turn is tantamount to power-law behaviour. The equivalence of statistical self-similarity and power-law behaviour is primarily a mathematical issue. A power law looks the same everywhere, i.e. if you take a small piece of a power-law tail and inflate it, it is identical with a larger piece of the initial curve. This is different, in particular, for exponential functions like the Gaussian, which drops sharply towards zero for small values already and then lies almost on the x-axis. The next point to be explained is the connection between statistical self-similarity and long-range correlations. For random processes like coin tossing there is no correlation between, in this case, two coin tosses. There is a fifty-fifty chance for either side in each toss. Even if you had heads ten times in a row, there is still a fifty percent chance for heads in the eleventh toss. And if you look at the probability distribution for many samples of 10 consecutive coin tosses you will get a completely different result than for samples of 100 consecutive coin tosses. The probability distributions for sequences of independent random variables are not self-similar, i.e. they have different statistical properties on different scales. This is very different, say, for (healthy) heartbeat intervals or stock prices. If there are days with drastic stock price movements there is a much higher chance for still more days with large changes in the immediate future than in quiet times (even though there is no correlation between the direction of these changes, i.e. up or down, which is the reason why it is not easy to exploit this knowledge). Thus changes of stock prices have a memory.

Another important and closely connected point is that for independent random processes (e.g. coin tosses) there is a negligible probability that very many subunits (e.g. individual tosses) all do the same, which would lead to extreme events like 100 consecutive times heads. If such an extreme events happens, there is either an external cause, e.g. a magnetic heads-detecting device, or the subunits most likely interacted with a coordinating effect. Thus statistical facts about a complex system can supply strong reasons for specific inferences about the existence or non-existence of underlying interactions between the system's parts with a collective effect.

Scientists in complex systems research make every effort to discover power-law and therefore scale-invariant relations because it has far-reaching implications for the behaviour of the system under investigation. Most importantly, under certain conditions, most microscopic details become irrelevant for the dynamics of the system on the macroscopic level. As one can learn from studies of so-called critical phenomena in statistical physics, the occurrence of scale-invariance and hence self-similarity is the 
deeper reason why diverse systems can exhibit very similar or even identical behaviour, a fact that physicists call 'universal behaviour'. 'Universality' in this sense can be explained via the method of renormalisation involving iterative course graining, which in turn would not be possible without self-similarity. Thus there is a direct road from power-law behaviour, scale-invariance and self-similarity to understanding why certain universal structural mechanisms can account for phenomena in physics as well as in economics. ${ }^{8}$ More specifically, power-law behaviour allows applying 'scaling methods', which were first devised in physics, in very different contexts such as economics.

In the context of dynamically complex systems, and also in my two examples in the next section, statistical self-similarity is of particular importance. Spatial self-similarity is relatively well-known from branching trees, snowflakes and coastlines which display spatial structures of the same type on small and large scales (see figure 1). The temporal kind of self-similarity shown in the right diagram is more abstract. It refers to the statistical properties of a temporal process, namely the probability distribution of the deviations of some quantity from one time step to another. For this reason one talks about 'statistical self-similarity'.

Self-Similar Structure

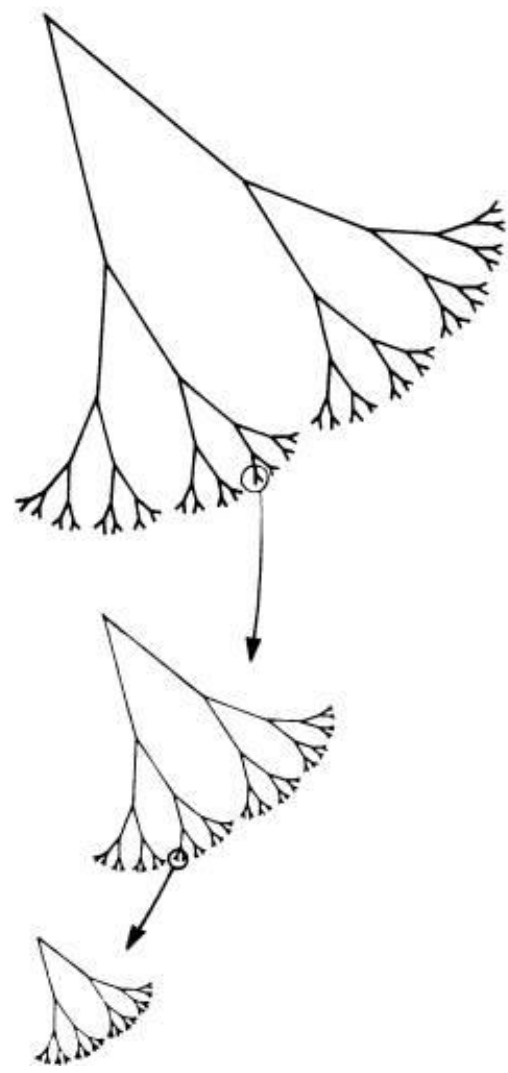

Self-Similar Dynamics $300 \mathrm{~min}$

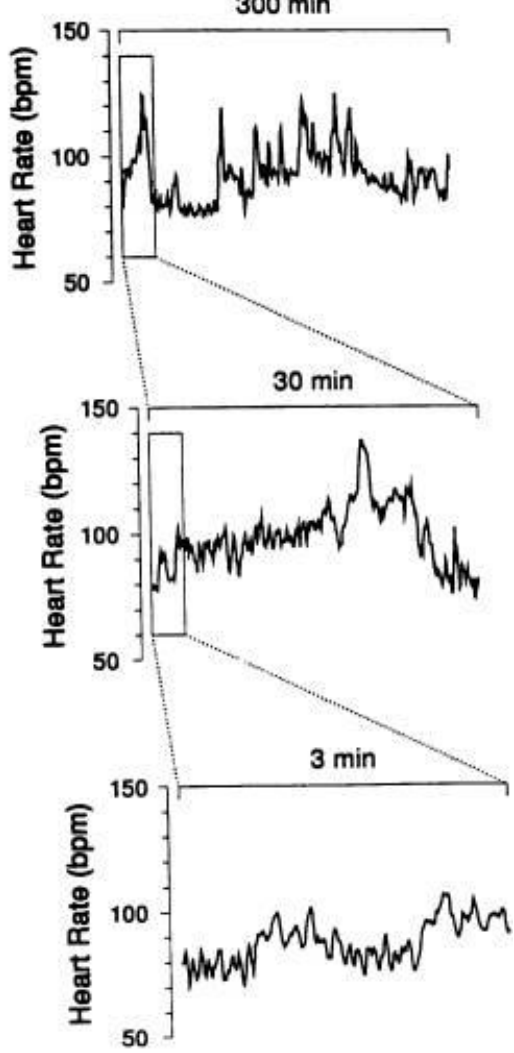

\footnotetext{
${ }^{7}$ See Binney et al. (1992) for a detailed account of the physical and Batterman (2002) of the philosophical perspective.

${ }^{8}$ Newman (2005) cautions that the mechanism discovered for critical phenomena is only one among various different mechanisms generating power-law behaviour.
} 
Figure 1. Schematic comparison of spatial (left diagram) and temporal (right diagram) self-similarity. In both cases, zooming repeatedly into parts of the initial structure/dynamics reveals structures/dynamics with similar or even identical characteristics as the initial one. Reprinted from Goldberger 2006 (will ask for permission).

One practically important aspect of scale-invariance stems from the fact that it is a symmetry principle. As in the well-known spatio-temporal cases, e.g. translational, rotational or Galilean invariance, the corresponding symmetry principles often allow for simple and elegant solutions of otherwise intractable problems. For instance, symmetry considerations often make it possible to derive important aspects of a system's dynamics without solving the underlying equations of motion. In other words, in certain important respects the dynamical behaviour of a system can be understood by abstract reasoning concerning its symmetries without any detailed knowledge about the behaviour of its fundamental constituents. The significance of these facts for econophysics, for instance, is straightforward. The application of physics to economical issues is, to a large extent, possible because financial market behaviour exhibits invariances that allow neglecting certain micro details, thus making way for analytical methods and explanatory models developed in physics, in order to understand the behaviour of systems in those special circumstances where, just as in financial markets, many micro details loose their relevance in a sharply specified sense.

In the following section I will exemplify the significance of scale-invariance in two different concrete contexts. More importantly, I will discuss the connection with mechanisms in dynamically complex systems. 


\section{On Heartbeat and Financial Market Crashes}

\subsection{Congestive Heart Failure}

Traditionally, cardiologists have described the normal heart beat activity as a regular sinus rhythm. However, in contrast to our subjective impression and to the traditional cardiologists' assumption, interbeat intervals normally fluctuate, even for individuals at rest, in a complex way, which appears to be erratic. The upper time series (a) in figure 2 shows the heart rate dynamics of a healthy person, while the lower one (b) the dynamics of a person with congestive heart failure.
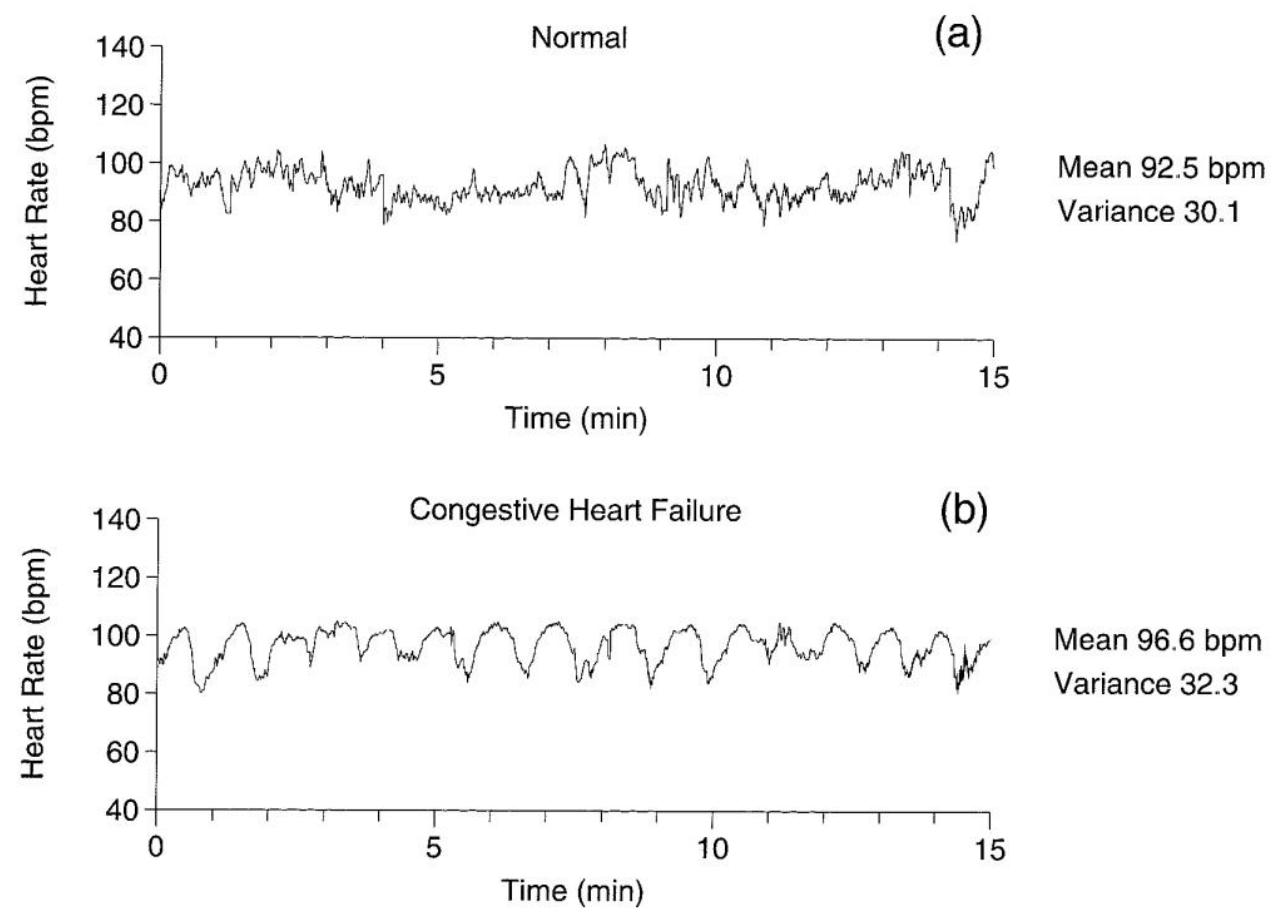

Fig. 2. Comparison of (a) healthy and (b) unhealthy heart rate dynamics. Contrary to one's expectation, healthy heart rate activity is far more complex, and seemingly erratic, than the relatively regular heart rate activity of unhealthy individuals. Only subtle data analysis is the first important step towards explaining this surprising difference. Reprinted from Ivanov et al. (2002) (will ask for permission).

Although the 'healthy dynamics' exhibits a far more complex pattern of variability than the 'unhealthy dynamics' with its rather periodic temporal structure, their mean values as well as their variances are almost identical. Thus the unexpectedly irregular behavior of heart beat activity defies conventional methods of analysis, which only work for stationary or 'well-behaved' time series. In order to analyse such data sets with fluctuations on multiple time scales one needs sophisticated techniques of 'fractal analysis', one of which is depicted in figure 3. 

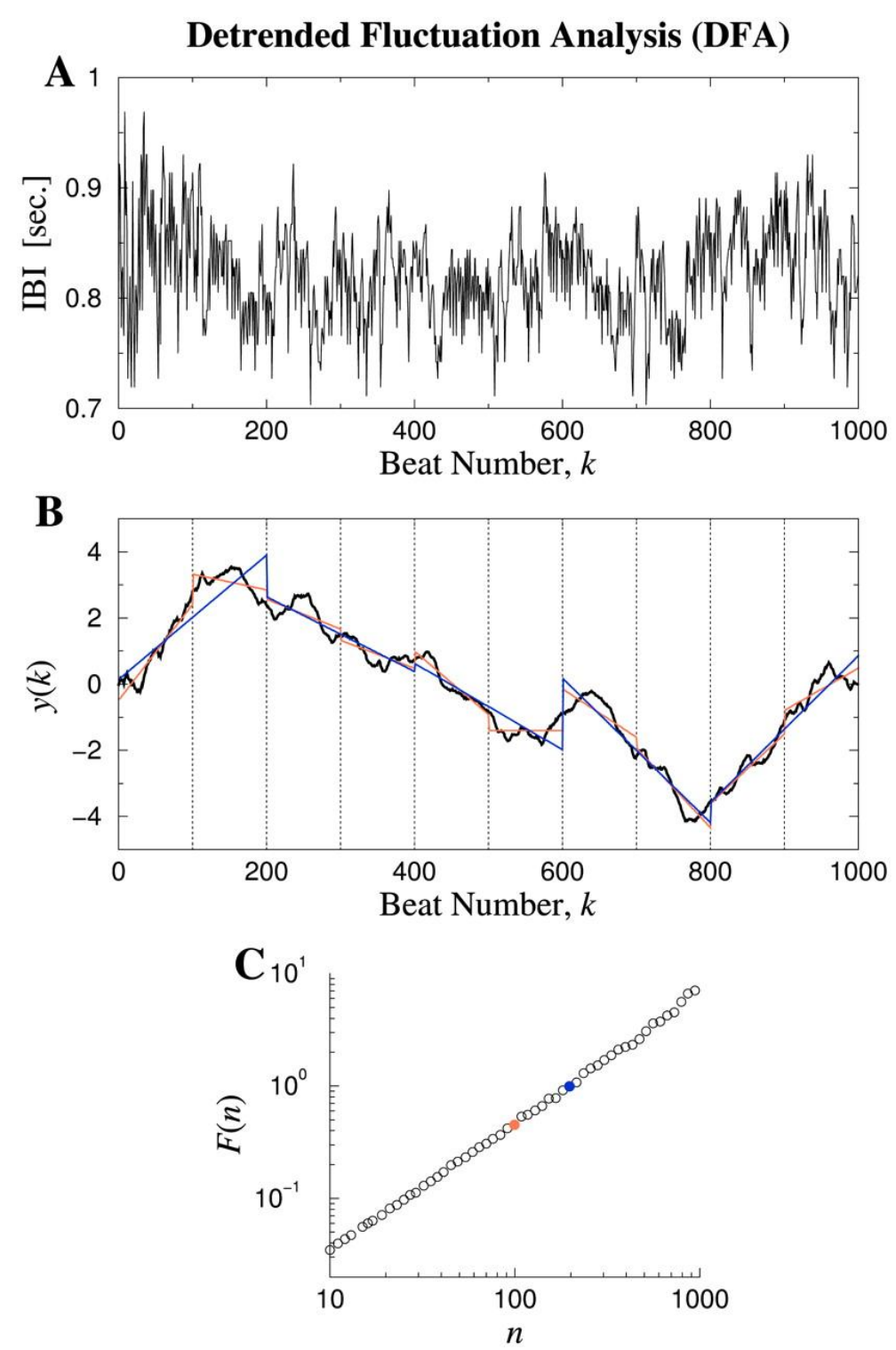

Figure 3. The method of detrended fluctuation analysis (DFA) allows uncovering hidden dynamical patterns, which are strong indicators for specific underlying mechanisms. The above diagrams depict the stepwise isolation of a power law in a seemingly erratic time series of heart beat (see main text for further explication). Reprinted from Goldberger et al. (2002) (will ask for permission).

The detrended fluctuation analysis, or short 'DFA method', is very useful in revealing to what extent there are so-called long-range correlations in a given nonstationary time series, where non-stationarity means that the statistical properties of the time series, such as the mean value, vary with time. ${ }^{9}$ In such cases one needs a sophisticated method since linear or higher order polynomial trends in the data often lead to the spurious detection of long-range correlations. In the first step of the DFA

\footnotetext{
${ }^{9}$ The DFA method was first presented in Peng et al. (1994) for the analysis of DNA nucleotides.
} 
method, applied to heart beat dynamics, a time series of interbeat intervals such as the one in diagram $\mathrm{A}$ of figure 3 (with $\mathrm{N}$ beats in total) is integrated, where the average interbeat interval $R R_{\text {ave }}$ is subtracted from each interbeat interval $R R(i)$, so that one gets $y(k)=\sum_{i=1}^{k}\left[R R(i)-R R_{\text {ave }}\right]$ as the integrated time series (see diagram B in figure 3 ). ${ }^{10}$ Note that if the interbeat intervals were all equal, then $R R(i)-R R_{\text {ave }}$ and thereby $y(k)$ would vanish and the DFA method would be redundant. Moreover, if the interbeat interval time series were stationary, i.e. if the interbeat intervals would randomly fluctuate around a constant mean value, then $y(k)$ would also vanish, again making the DFA method redundant. Thus, a non-vanishing integrated time series $y(k)$ reveals fluctuations that are not evenly distributed around some mean value, i.e. it makes the non-stationarity of the time series visible and quantifiable. In particular, it allows detecting whether the heart beat intervals temporarily tend into one direction, e.g. becoming either shorter or longer. That this happens is well-known to everyone by firsthand experience.

The DFA method allows investigators to extract and put aside these 'trends' (therefore the name 'detrended fluctuation analysis') in order to get an undisturbed view into hidden statistical patterns which may indicate certain underlying processes. This removal of trends is done in the following way. The integrated time series gets divided into equal boxes and in each box a 'least squares line' is fitted to the data, which is taken to represent the trend in that box (see diagram B in figure 3). In the next step, the integrated time series gets detrended by subtracting the local trend in each box of the chosen size $n$. Eventually, one calculates the root-mean-square fluctuation of the integrated and detrended time series and repeats the same procedure over all time scales, i.e. all box sizes, in order to determine the relationship between the average fluctuation $F(n)$ and the respective box size $n$. If, on a double logarithmic plot one finds a linear relationship, this indicates the presence of power law scaling and thereby of a fractal structure of the time series. ${ }^{11}$ In this case the fluctuations can be characterized by a scaling exponent, which is the slope of the line in diagram $\mathrm{C}$ of figure 3 . At this stage it may be helpful to consult section 2.2 again, where I already explained the more general implications of these issues.

Thus using the DFA method one finds that the fluctuations of the healthy (but not the unhealthy) heart rate dynamics are statistically self-similar, i.e. the statistical properties of heart rate fluctuations are identical on different time scales. Thus healthy heart rate regulation generates statistically self-similar fluctuations, which is tantamount to long-range correlations in the time series. ${ }^{12}$ Goldberger, one of pioneers of 'fractal physiology', offers the following conclusion:

"A defining feature of healthy function is adaptability, the capacity to respond to often unpredictable stimuli. [...] Fractal physiology, exemplified by long-range correlations in heartbeat and breathing dynamics, may be adaptive for at least two

\footnotetext{
10 'RR' stands for Scipione Riva-Rocci, who invented the traditional procedure for measuring blood pressure.

${ }^{11}$ In a double logarithmic plot, i.e. if the logarithm of both the $x$ - and the $y$-coordinate of a function $y(x)$ is taken, a power law $y(x)=x^{\text {a }}$ becomes a straight line.

${ }^{12}$ See Binney et al. (1992) for the connection between the statistical self-similarity of fluctuations and the existence of long-range correlations.
} 
reasons [...]: (1) long-range correlations serve as an organizing mechanism [my emphasis] for highly complex processes that generate fluctuations across a wide range of time scales and (2) the absence of a characteristic scale may inhibit the emergence of very periodic behaviors that greatly narrow system responsiveness. This hypothesis is supported by findings from life-threatening conditions, such as chronic heart failure where the breakdown of fractal correlations is often accompanied by the emergence of a dominant mode [...] The paradoxical appearance of highly ordered dynamics with pathologic states ("disorders") exemplifies the concept of complexity loss (decomplexification) in aging and disease $[. .$.$] . Physiologic stability appears to relate in part to complex patterns of$ variability that incorporate long-range correlations [...] The opposite of a fractal (scale-free) system [...] is one dominated by a characteristic frequency [...]." (Goldberger (2006))

Goldberger directly (although tentatively) interprets the long-range correlations in the time series of healthy heart beat, as found by means of the DFA method, as an 'organizing mechanism' that helps to generate fluctuations across many time scales, which secure the responsiveness of a healthy heart to unpredictable influences.

What is it that justifies Goldberger's (and other's) hypothesis that a mechanism has been identified? The first, very important point is the robustness of the statistical characteristics that the DFA method allows one to identify in healthy heartbeat dynamics. For different healthy individuals in the same context (e.g. sleep or wake phase) one always finds the same characteristics. Second, there has been some transfer of knowledge from structurally similar situations. ${ }^{13}$ In particular there is a large body of experience with complexly fluctuating quantities in condensed matter physics. From these cases one knows that certain statistical characteristics of fluctuations imply longrange correlations, which arise purely endogenously by the non-linear interaction of the system's subunits in the absence of any coordinating external force. If this inference is justified, it suggests an (endogenous) mechanism that leads to correlations between (spatially or temporally) distant subunits of the system. As a third point, a mechanistic interpretation of the results of data analysis such as Goldberger's always rests on specific contextual knowledge. For instance, one needs to know, I want to argue, that the responsiveness of heartbeat to unpredictable influences is an important ability of healthy individuals, so that one can surmise that there is some corresponding mechanism. Despite of these points in favour of Goldberger's mechanistic interpretation the question is not yet conclusively answered whether he is in fact justified in claiming the discovery of a mechanism. In my evaluation such an interpretation is not sufficiently grounded as long as not even a sketch has been supplied as to how the component parts may, by their compositional and interactive organisation, generate the phenomenon of interest. I will come back to this point in the final discussion. Before doing so I want to present another example which is closely related to the first one in some structural aspects, whereas materially we will be concerned with a completely different subject

\footnotetext{
${ }^{13}$ The following quote by condensed matter physicists indicates the fundamental significance of this point: "What makes continuous phase changes especially interesting is the scale-freedom of the fluctuations at $[\ldots]$. Not only is the creation of long-range structure by short-range inter-molecular forces intriguing, but any example of scale-freedom is worthy of close examination since this phenomenon occurs in several physical systems that are inadequately understood." (Binney et al. 1992: 30)
} 
matter. Moreover, the situation will be different regarding the legitimacy of talking about mechanisms.

\subsection{Financial Markets}

My second candidate for a mechanism in a dynamically complex system occurs in socalled microscopic models of financial markets, within the context of econophysics (see above). Since mechanisms are always relative to some behaviour that is to be explained, it is necessary to describe what it is that econophysicists want to explain and why they rate complex systems theories, as developed in condensed matter physics, as the appropriate framework for this end. The endogenous formation of financial market crashes, i.e. without any particular external causes, is one particularly well-known example for a characteristic dynamical pattern that calls for an explanation. Put more generally, financial markets experience far more large changes and extreme events, like crashes and bubbles, than one would traditionally expect for random processes, such as Brownian motion. Econophysics often talk about 'fat tails' in the probability distributions for price changes in assets like stocks or commodities, since the corresponding functions stay way above the $\mathrm{x}$-axis much longer than in Gaussian probability distributions for random variables like body size or IQ. Another closely connected example for a characteristics dynamical feature in financial market that calls for an explanation is the so-called volatility clustering, i.e. the tendency of quiet and turbulent market periods to cluster together in packages. These characteristics of financial markets indicate that the interaction between market participants is of crucial significance. That is, the best explanation for the high probability of extreme events in financial markets involves the assumption that financial markets are complex systems with non-linearly interacting constituents, just as many other composite systems that show a similar tendency for the endogenous formation of extreme events in the absence of any dramatic external causes. ${ }^{14}$

One main research activity in econophysics is the construction of so-called microscopic models of financial markets ${ }^{15}$ that reproduce the observed statistical features of market movements (e.g. fat tailed return distributions, clustered volatility, crashes) by employing or inventing highly simplified models with large numbers of agents (market participants). ${ }^{16}$ Thereby one tries to understand the main statistical characteristics of observed probability distributions in terms of underlying random processes, e.g. random walk. The relevant parts of physics that are used to build microscopic models of financial markets are usually models and methods from condensed matter physics and statistical physics. Microscopic models of financial markets are highly idealized as compared to what they are meant to model. Often all agents have identical properties or there are very few subgroups. Another option is to

\footnotetext{
${ }^{14}$ See Mantegna and Stanley (2000: 5), Sornette (2003: 15) and Schweitzer (2003, section 1.1).

${ }^{15}$ In the last decade economists and physicists investigated various microscopic (or 'agent-based') models of financial markets, for instance the Kim-Markowitz, the Levy-Levy-Solomon, the Cont-Bouchaud, the Solomon-Weisbuch, the Lux-Marchesi, the Donangelo-Sneppen and the Solomon-Levy-Huang model. See Samanidou et al. (2007) for a review of these models.

${ }^{16}$ See Voit (2001) and Johnson et al.( 2003) as well as Casti (1997) for the wider background.
} 
have a set of agents with random variations. The interaction between agents is usually modelled as extremely simple, like 'do what your nearest neighbour does'.

A paradigm case of a microscopic model for financial markets arose from the collaboration of the economist T. Lux and the physicist M. Marchesi. ${ }^{17}$ Their stochastic multi-agent model rests on the empirical fact that the universal characteristics of price change statistics (fat tails, clustered volatility) are structurally similar to scaling laws in physics. In physics, scaling laws arise from the interaction of a large number of interacting units, e.g. particles, where most microscopic details are irrelevant. This structural similarity of observed phenomena in physics and finance suggests an equally similar explanation. In the Lux/Marchesi model there are two types of traders, 'fundamentalists' and 'noise traders' (or 'chartists'). ${ }^{18}$ Whereas fundamentalists are rational traders who base their action on the comparison of the fundamental value $p_{f}$ of the traded asset (e.g. stocks, bonds or currencies) and the actual market price $p$, the behaviour of noise traders only depends on the current price trend and the opinion of other traders. A crucial feature of the setting used by Lux and Marchesi refers to the dynamics for the fundamental value $p_{f}$, more precisely its relative (logarithmic) changes between two time steps, which are assumed to be Gaussian random variables. This assumption is decisive for the Lux/Marchesi approach because it means that changes of $p_{f}$ cannot be the reason for the typical statistical features of financial assets like fat tails and clustered volatility, which the model in meant to reproduce in its dynamics. Figure 4 shows the result of one 'computer simulation run'.

a

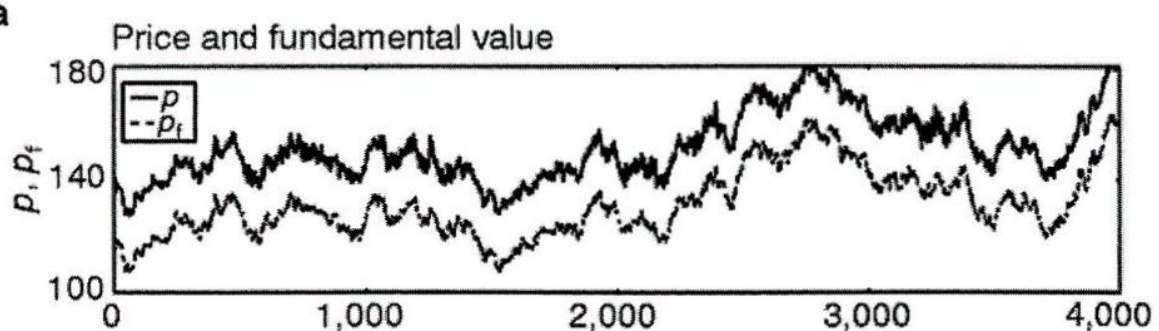

b

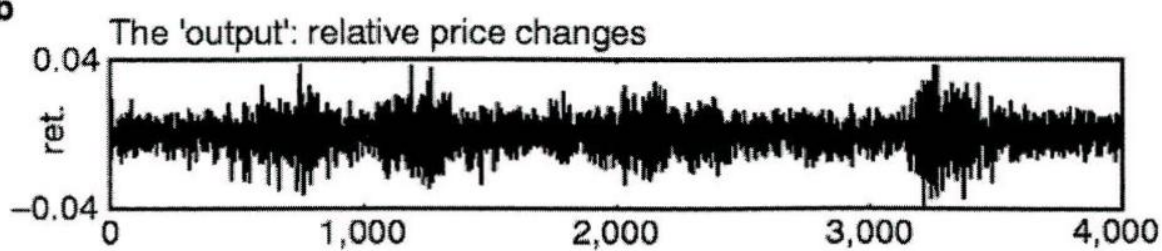

c

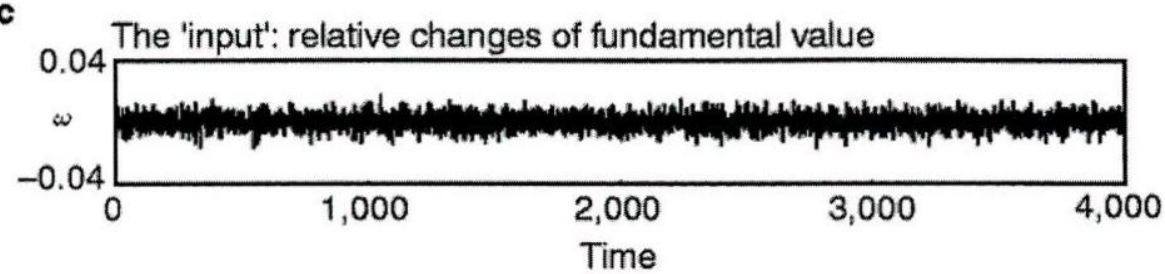

\footnotetext{
${ }^{17}$ See Lux and Marchesi (1999).

${ }^{18}$ The coinage of and the distinction between 'fundamentalists' and 'noise traders' is not due to Lux and Marchesi, but is established in economics.
} 
Figure 4. Result of a typical simulation run for a stochastic multi-agent model of a financial market. Most importantly, in comparison to the assumed input series in diagram $\mathrm{c}$, the resulting time series in diagram $\mathrm{b}$ shows far more large changes as well as packages or clusters of very volatile asset prices. Both of these characteristics must have been produced purely endogenously by the interaction of the market participants. (Reprinted from Lux and Marchesi 1999, with permission by Nature.)

The intuitively most compelling impression of the result can be gained by comparing the time developments of the market price $p$ (upper curve) and the fundamental value $p_{f}$ (lower curve), first, with each other, and, second, with respect to their statistical properties. The most interesting statistical property is the frequency of price changes from one time step to another. The crucial point of the result is that the time developments of the market price and the fundamental value are very similar whereas, at first sight surprisingly, their statistical properties differ remarkably. The two lower diagrams show the relative price changes which are extracted from the time developments shown in the first diagram. Only after this extraction does the difference between the distribution of changes of the market price and the fundamental value become visible. Although the market price tracks the fundamental value in average it deviates significantly on a short time scale, allowing for the typical 'extreme events' and the clusters of high volatility which are observed in real markets. Lux and Marchesi conclude that the market is efficient in the sense that the market price follows the fundamental value. This does not apply to the short term, however, where the relative changes of the market price deviate from the normal distribution, which was assumed for the relative changes of the fundamental value.

In their analysis Lux and Marchesi also use the DFA method which I introduced above for the investigation of heart rate dynamics. The analysis of the scaling properties (in particular the extraction of critical exponents) shows that the exponents for the exogenous input series (i.e. the random changes of the fundamental price $p_{f}$ ) do not allow for fluctuations of the order of empirically observed price changes. Lux and Marchesi show that the emergence of a power-law distribution of price changes is produced by changes from quiet to volatile periods, which are due to transitions of agents from one group to another, more precisely from fundamentalists to noise traders. This behaviour, which is sometimes called 'switching', will play an important role in my own analysis of how Lux and Marchesi contribute to the scientific explanation of financial market behaviour. Another result of Lux and Marchesi is that a system looses its stability when the number of noise traders exceeds a certain critical value, and they observed so-called 'on-off intermittency', i.e. the fact that instabilities are recurrent but only temporary. Eventually, it should be stressed that the qualitative results of Lux and Marchesi are very robust since temporary instability (high volatility) occurs for a wide range of parameter values.

Again, let me ask the question, whether it is justified to say that certain mechanisms have been found? Similarly as in the heart beat example, the first important point in 
favour of talking about mechanisms is the robustness of the statistical characteristics which have been identified in financial market dynamics. For Lux and Marchesi's microscopic models of a financial market, fat-tailed probability distributions and volatility clustering are stable characteristics of their computer simulations that do not depend on any particular parameter values or any particular initial configuration. And the other two points in my evaluation of the heart beat example, namely about the transfer of knowledge from structurally similar situations as well as about additional contextual knowledge, are also in place. But in the financial market case, compared to the heart beat example, much more has been said about component parts (the agents) and their compositional and interactive organisation (different groups of traders, certain rules for buying and selling behaviour). And one also gets a clearer picture about how the compositional and interactive organisation of the component parts can generate the phenomenon of interest. Nevertheless, this is still much less than in classical mechanisms. In comparison to the well-known multifacetedness and irrationality of real financial markets the described model of a financial market seems ludicrously simple. This observation seems a crucial point to me to which I will come back in my final evaluation.

Summing up, for both the heart beat and the financial market example, one strong point in favour of talk of mechanisms is the fact that certain subtle statistical characteristics of the respective dynamics can be shown to arise in a robust way. However, although the reference to the level of component parts and their compositional and interactive organisation also remains more or less vague in both cases, when compared to the complexity of the phenomena that are investigated, the sketch of the 'microscopic' processes are far more convincing in the financial market case. In the next section I will argue that this difference is in fact crucial regarding claims about mechanistic explanations.

\section{Complex Systems and Mechanical Philosophy}

How could the potential mechanisms in dynamically complex systems be incorporated into the program of mechanical philosophy? Thorough answers are hard to be found. A promising and very recent answer is presented in Bechtel and Abrahamsen (forthcoming). Although Bechtel and Abrahamsen come closest to how I see the matter there remain some diverging points. One of their central claims is that despite of terminological differences ${ }^{19}$ there is a consensus about the crucial steps of what they call a 'basic mechanistic explanation', namely "(1) the identification of the working parts of the mechanism, (2) the determination of the operations they perform, and (3) an account of how the parts and operations are organized so that, under specific contextual conditions, the mechanism realizes the phenomenon of interest." 20 Bechtel and Abrahamsen concede that, as it stands, the basic notion of mechanistic explanation is too limited to account for the insights into the complex dynamics of biological

\footnotetext{
${ }^{19}$ For instance, Machamer, Darden and Craver (2000) stress the dichotomy of 'entities' and 'activities', whereas Glennan $(1996,2002)$ emphasizes the interaction between the parts of a mechanism.

${ }^{20}$ See Bechtel and Abrahamsen (2011), all following quotes come from this paper, unless otherwise stated.
} 
mechanisms which have been achieved by complexity theories. However, they argue, there is no need to supplant the mechanistic philosophy of science by the new paradigm of complex systems modelling since it is possible and preferable to correct and thus modify the mechanistic approach appropriately by incorporating the relevant ideas of complex systems theories. The resulting notion of what they call 'dynamic mechanistic explanation' is meant to recognize the "previously neglected temporal dynamics and the implications for our understanding of how operations are orchestrated in real time" or in other words, the "temporal dynamics that orchestrate the functioning of biological mechanisms."

I agree with Bechtel and Abrahamsen that the idea of mechanistic explanations should not be given up in favour of complex systems theories since many tools and concepts of complex systems theories can and should be integrated into the more comprehensive conception of mechanistic explanations. However, I think the phenomenon of dynamical complexity that I am focussing on cannot be fitted into the existing theories of mechanisms by adding insights into the complexity of the dynamics of mechanisms, by recognizing how operations are "orchestrated in real time", to use a phrase by Bechtel and Abrahamsen. Instead, I claim that in the case of what I call dynamically complex mechanisms, understanding the robust dynamical patterns of the system is in fact the core task of the researcher whereas the identification of parts, operations, and their organisation looses much weight, although it is not completely lost. Detailed analyses of the parts and the operations of these parts and the organisation, including the detailed organisation of their interactions, are of minor interest, since they have, to a surprisingly high degree, no effect on the dynamical characteristics of interest. They constitute the set-up but nothing much is understood if only the parts, their interaction behaviour and the initial arrangement of the whole system are specified. Rather, one has to identify the dynamical patterns of the compound system of interacting parts. When complex systems researchers try to understand by which mechanisms these dynamical patterns are generated they usually make certain assumptions about the parts of the complex system, their interaction behaviour, and the basic arrangement of the whole system so that it displays the phenomenon one wants to be explained. But finding an appropriate set of assumptions as such by no means exhausts the identification and understanding of mechanisms in dynamically complex systems. Moreover, often the parts in dynamically complex systems can change their nature completely while the complex system evolves in time, as one can quickly see in the econophysics example. Traders can switch from one group to another, which is in fact a crucial characteristic of the model. But if the parts are taken to be identified by their behaviour, then one is forced to say that that there isn't even a stable set of entities in a dynamically complex system.

So what should we conclude concerning the question how complex systems research and mechanical philosophy are related? Bechtel and Abrahamsen offer the following conclusion:

"Dynamic mechanistic explanation stands in contrast not only to purely mechanistic explanation but also to theoretical inquiries that emphasize complex dynamics in living systems conceived abstractly - at best neglecting but in some cases explicitly rejecting the mechanistic project. Artificial life research, for 
example, is conducted on a plane removed from research on actual biological mechanisms. While accounts oriented purely to complexity or dynamics can make unique and valuable contributions, they provide no understanding of how the dynamic relations are actually realized in living systems if they do not get anchored to component parts and operations of actual mechanisms. That is, they are empty. We contend that complexity and dynamical systems theory find their best use as tools in a more integrated endeavor." (Bechtel and Abrahamsen (forthcoming))

Again, I agree with Bechtel and Abrahamsen that an understanding of complex dynamics does and should not supplant mechanistic explanations. However, as I have shown in the two examples above, mechanisms in dynamically complex systems are not appropriately covered by the standard notions of mechanisms. To some extent this judgement is in agreement with Bechtel and Abrahamsen, but a closer look reveals important differences. In order to see that it is helpful to consult two other writings by Bechtel, together with Richardson:

\begin{abstract}
"The interactions between subsystems become increasingly important as the units engage in more complex modes of interaction, such as [...][different] kinds of feedback [...]. [...] Thus, emergence is a consequence of complex interaction. Different models are needed to characterize the interactions between the components in a complexly organized system than are needed to characterize the behavior of the independent components. With emergent phenomena, it is the interactive organization [my emphasis, M.K.], rather than the component behaviour, that is the critical explanatory feature." (Bechtel and Richardson (1992: 285))
\end{abstract}

In what Bechtel and Richardson (1993) call 'integrated systems' the behaviour of the whole system is mostly determined by the (nonlinear) interaction of its components. And in still extremer cases "the activities of the parts seem to be different in kind from, and so far simpler than those performed by the whole. The parts can be so simple, in fact, that they do not seem to contribute anything of interest to understanding the behavior of the whole; in some cases it is possible to destroy or disable much of the system without significantly affecting performance." 21 While Bechtel and Richardson think of network models of cognition something similar applies to microscopic models of financial markets, for instance. Here the statistical patterns are not altered by adding or removing however many specific traders, as long as we are still dealing with a large number of nonlinearly interacting heterogeneous subunits.

For a better understanding of mechanisms in dynamically complex systems I think the eventual shift away from classical mechanistic thinking is not radical enough as long as the basic idea remains that we have a certain number of working parts, say, A, B, C, D, E, F and G, each of which can perform certain operations and which interact with each other in a nonlinear way thus giving rise, for instance, to self-sustained oscillations like in the circadian rhythm. I do not intend to reject this analysis in the cases Bechtel in particular is studying but I want to point out that not all explanations in terms of nonlinear mechanisms are appropriately represented by this description. In those dynamically complex systems I am focussing on in this paper it is inappropriate to emphasize the identification of particular working parts and certain operations they

\footnotetext{
${ }^{21}$ Bechtel and Richardson (1993: 202f).
} 
perform. The kind of nonlinear mechanisms I scrutinize work largely irrespective of the detailed individual natures of the subunits that are involved and their initial compositional as well as their detailed interactive organisation in the whole system.

Since it is apparent from complex systems that are microscopically well-understood that most micro details can be irrelevant (relative to one's explanatory target), it is only consistent that detailed investigations about the organisation of mechanisms become less important in the case of dynamically complex systems. Instead, the focus is shifted towards studying the dynamics. For instance, it is of great interest, under which conditions the dynamics is robust and in which cases instabilities occur. However, if the attention is exclusively directed towards analysing the (statistical) characteristics of the dynamics, then the point is reached where, in my judgement, the researchers' use of the term 'mechanism' is no longer justified since it transgresses the limits of even the most minimal notion of mechanisms. For example, one can sometimes find talk about 'fractal mechanisms', although fractality is a feature that only refers to the statistics of time series. Without any further knowledge, all one is warranted to say in this case is that the statistical characteristics one has found indicate certain underlying mechanisms. But no mechanism has been identified unless at least some indication has been given about how an interaction of subunits may be involved to generate the phenomenon of interest. The inevitably vague qualification 'at least some indication' is of crucial importance. Requiring more than that would, in my view, make too many complex systems studies non-explanatory. And more importantly, the valuable explanatory perspective of complex systems theories would be diminished if its structural focus were given up, where specific material details are deliberately faded out.

The difficulties in fitting the potential mechanisms in dynamically complex systems into the existing notions of mechanisms prompts the question whether one should talk about mechanisms at all in this case. Is there any need or at least are there good reasons for construing explanations for the behaviour of dynamically complex systems in terms of mechanisms? A first strong indication that one should indeed be talking about mechanisms is that the term is ubiquitous in actual analyses of complex systems. For instance, there is an extensive discussion about the "mechanisms" that generate power law behaviour, which is closely connected with scale-invariance (see above). ${ }^{22}$ Although in some cases the term 'mechanism' seems inappropriate, widespread terminology among scientists should be taken seriously. Nevertheless, this point alone does not yield a conclusive justification. A closer look at the econophysics example from above already provides a firmer basis. In the actual scientific practice of econophysics there are clearly two different areas of research. On the one hand we have statistical analyses which provide the systematic identification of explananda. But on the other hand, many investigations are concerned with the formulation of explanatory microscopic models, i.e. models that reproduce or 'generate' the observed phenomena, in particular their statistical characteristics. And it seems that the underlying conception of explanation is mechanistic, partly because there are no established laws on the micro level that would allow invoking the covering law model, for instance. Moreover, to a certain degree, interdisciplinary approaches such as econophysics rest on the transfer of

${ }^{22}$ See, for instance, Newman (2005) and Sornette (2006: chapter 14). 
mechanistic models from one scientific field to another, e.g. from condensed matter physics to economics. Eventually, there are two further reasons why a construal of explanations in complex systems theories in terms of mechanisms is attractive. First, it supplies important means in order to answer questions concerning the reducibility of complex systems behaviour. Naturally, this point is only attractive if one is interested in micro reductions. Second, mechanistic explanations are arguably the best way towards finding effective interventions and many investigations in complex systems research have this goal.

\section{Towards a More Structural Notion of Mechanisms}

Structural explanations that rest, for instance, on basic symmetries independently of any particular ontology have a long and successful history in physics. Elementary particle physics lives on considerations where symmetry principles are the cornerstones. With the advent of the statistical mechanics of complex systems and modern computing, structural explanations spread into various fields far beyond fundamental physics, at first within physics, eventually into almost each science. Today, the same analytical techniques, concepts, models and explanatory strategies are applied across radically different sciences such as physics, biology, economics and social science. Apparently, the success of this transfer does not rest on a common ontology - unless one wants to reify structures, which I do not advocate. In a sense these sciences have the same underlying ontology since, for instance, markets traders, human hearts and ocean waves ultimately all consist of elementary particles. But this common fundamental ontology is not the reason why the same explanatory strategies can successfully be applied. In the context of complex systems theories the reason is the observable fact that there are structural similarities in the dynamics of compound systems with completely different kinds of subunits. These structural similarities can be classified in terms of certain dynamical patterns that can in turn be represented and discriminated in a mathematically precise and subtle way.

A concrete example for structural similarities in the dynamics of extremely diverse complex systems is probably more helpful than 1000 words. Ferromagnets have the surprising ability to form a macroscopic magnetization if the temperature falls below a certain threshold. Detailed analyses revealed, roughly sketched, that the underlying mechanism involves the endogenous, i.e. not externally coordinated, parallel alignment of neighbouring dipoles (spins) across the whole piece of matter, whereas the dipoles are irregularly oriented for higher temperatures. Physicists talk of a phase transition, which results in long-range correlations of otherwise uncorrelated dipoles (and of course in self-similarity, power laws, and all that). Reasoning in structural analogies helped enormously to understand that something very similar happens in financial markets. Here as well it is the mutual interaction between traders (analogous to dipoles) and their ability to change the neighbour's trading behaviour (analogous to the orientation of the dipoles) that is crucial for understanding the endogenous formation of large changes and even comprehensively collective behaviour (e.g. financial market crashes). Once this structural analogy is understood it allows for far-reaching explanatorily valuable conclusions without the need for detailed analyses of the micro 
details. What still needs to be done, however, is a convincing proof that the analogy actually holds. That is, sufficient grounding in the actual situation of financial markets must be supplied, for otherwise one just has an interesting speculation. But insisting on a complete specification of the microscopic situation would spoil the explanatory efficacy of this approach, since one of its crucial characteristics is the insight into the irrelevance of most micro details.

Today, complex systems with large numbers of nonlinearly interacting subunits have a similar significance as analytically tractable systems in the past. The behaviour of complex systems is much harder to understand and to predict than the behaviour of simpler classical systems. Nevertheless, for good reasons complex systems theorists firmly believe that-bearing in mind the much higher complexity of the subject matter - they can do more than just describe similarities of dynamical patterns. For instance, one can show under which conditions the statistical characteristics of dynamical patterns are robust and how these patterns arise on the basis of nonlinear interactions of subunits - subunits that need not be described more than in a rough structural way. Moreover, in some cases it can precisely be said at which point a system may loose its stability. This is less than in the classical cases since the further development cannot be accurately predicted, but still something explanatorily helpful can be said, e.g. for purposes of intervention.

Summing up one can say that complex systems theories can contribute substantially to the explanation of when and why certain structural dynamical patterns ${ }^{23}$ are generated in a robust way by the nonlinear interaction of the system's parts, even if these parts and their compositional and interactive organisation in the whole system are only roughly sketched. Therefore, I think it is justified to say that complex systems theories supply mechanistic explanations, provided a sufficient grounding in concrete interacting parts is supplied. In many cases, more detailed and concrete grounding may be desirable, but still a large number of cases will remain, where more details will be very hard to supply without deteriorating the explanatory efficacy. However, if one is willing to follow this step, the notion of mechanisms must be modified or adapted in a rather drastic way. Even the more sophisticated gloss that the understanding of mechanisms comprises the identification of parts, of the input-output behaviours of these parts and how the compositional and interactive organisation can bring about the phenomenon of interest has an inappropriate focus in the case of dynamically complex systems. Here, the emphasis lies not on the identification of material parts, their detailed behaviours and the initial set-up of the whole system but on identifying the structural conditions for the robust generation of characteristic dynamical patterns. To this end, a very simple description of the lower-level organisation can be sufficient for a mechanistic explanation, and sometimes even the best one can do.

Acknowledgement: I wish to express my gratitude to William Bechtel, Carl Craver, Stuart Glennan, Manfred Stöckler and two anonymous referees for very helpful comments on an earlier draft of this paper.

\footnotetext{
${ }^{23}$ By 'structural dynamical patterns' I mean patterns independently of any particular ontology.
} 


\section{References}

Batterman, R. W. (2002): The Devil in the Details, Oxford et al.: Oxford University Press.

Bechtel, W., and A. Abrahamsen (2005): Explanation: A mechanist alternative. Studies in History and Philosophy of Biological and Biomedical Sciences, 36: 421-441.

Bechtel, W., and A. Abrahamsen (2011): Complex biological mechanisms: Cyclic, oscillatory, and autonomous. In Collier, J., and C.A. Hooker (eds.): Philosophy of Complex Systems. Handbook of the Philosophy of Science, Volume 10. New York: Elsevier.

Bechtel, W. and R.C. Richardson (1992): Emergent phenomena and complex systems In Beckermann, A., Flohr, H., and J. Kim (eds.): Emergence or Reduction? - Essays on the Prospects of Nonreductive Physicalism, Berlin, New York: Walter de Gruyter.

Bechtel, W., and R. C. Richardson (1993): Discovering Complexity: Decomposition and Localization as Strategies in Scientific Research, Princeton: Princeton University Press.

Binney, J. J., Dowrick, N. J., Fisher, A. J. and M. E. J. Newman (1992): The Theory of Critical Phenomena: An Introduction to the Renormalization Group, Oxford: Clarendon Press.

Casti, J. L. (1997): Would-Be Worlds - How Simulation is Changing the Frontiers of Science, New York et. al.: John Wiley \& Sons.

Érdi, P. (2007): Complexity Explained, Springer.

Glennan, S. S. (1996): Mechanisms and the nature of causation, Erkenntnis 44: 49-71.

Glennan, S. S. (2002): Rethinking mechanistic explanation, Philosophy of Science 69: S342-S353.

Goldberger, A.L. (2006): Giles F. Filley Lecture. Complex systems. Proc. Am. Thorac. Soc. 3: 467-72.

Goldberger, A.L., Amaral, L.A.N., Hausdorff, J.M., Ivanov, P.Ch., Peng C.K., and H.E. Stanley (2002): Fractal dynamics in physiology: alterations with disease and aging. Proceedings of the National Academy of Sciences of the United States of America, 99: 2466-2472.

Hüttemann, A. (2004): What's Wrong With Microphysicalism? London, New York: Routledge.

Hüttemann, A., and O. Terzidis (2000): Emergence in Physics, International Studies in the Philosophy of Science 14: 267-281.

Ivanov, P.Ch., Goldberger, A.L., and H.E. Stanley (2002): Fractal and Multifractal Approaches in Physiology. In Bunde, A., Kropp, J., und H.J. Schellnhuber (ed..): The Science of Disaster: Market Crashes, Heart Attacks, and Climate Disruptions, Berlin: Springer.

Ivanov, P.Ch., Hu, K., Hilton, M.F., Shea, S.A., and H.E. Stanley (2007): Endogenous circadian rhythm in human motor activity uncoupled from circadian influences on cardiac dynamics. Proc. Natl. Acad. Sci. USA 104[52]: 20702-20707.

Johnson, N. F., Jefferies, P., and P. M. Hui (2003): Financial Market Complexity: What Physics Can Tell Us about Market Behaviour, Oxford: Oxford University Press.

Lux, T., and M. Marchesi (1999): Scaling and criticality in a stochastic multi-agent model of a financial market, Nature 397: 498-500. 
Lux, T., and M. Marchesi (2000): Volatility clustering in financial markets: A microsimulation of interacting agents, International Journal of Theoretical \& Applied Finance 3: 675-702.

Machamer, P., Darden, L., and C. Craver (2000): Thinking about mechanisms, Philosophy of Science 67: 1-25.

Mantegna, R. N., and H. E. Stanley (2000): An Introduction to Econophysics: Correlations and Complexity in Finance, Cambridge et al.: Cambridge University Press.

Newman, M. E. J. (2005): Power laws, Pareto distributions and Zipf's law. Contemporary Physics 46: 323-351

Peng, C.K., Buldyrev, S.V., Havlin, S., Simons, M., Stanley, H.E., and A.L. Goldberger (1994): Mosaic organization of DNA nucleotides. Phys. Rev. E 49: 1685-1689.

Peng, C.K., Havlin, S., Stanley, H.E., and A.L. Goldberger (1995): Quantification of Scaling Exponents and Crossover Phenomena in Nonstationary Heartbeat Time Series [Proc. NATO Dynamical Disease Conference], edited by L. Glass, Chaos 5: 82-87.

Samanidou, E., Zschischang, E., Stauffer, D., and T. Lux (2007): Agent-based models of financial markets, Reports on Progress in Physics 70: 409-450.

Sornette, D. (2003): Why Stock Markets Crash: Critical Events in Complex Financial Systems, Princeton and Oxford: Princeton University Press.Sornette, D. (2006): Critical Phenomena in Natural Sciences - Chaos Fractals, Selforganization and Disorder: Concepts and Tools, Berlin and Heidelberg: Springer.

Sterman, J. (2000): Business Dynamics: Systems Thinking and Modeling for a Complex World. Irwin McGraw-Hill.

Voit, J. (2001): The Statistical Mechanics of Financial Markets, Berlin, Heidelberg, New York: Springer.

Woodward, J. (2003): Making Things Happen - A Theory of Causal Explanation. Oxford: Oxford University Press.

Zvelebil, M.J., and J.O. Baum (2007): Understanding Bioinformatics. Garland Science. 